Case report

Perinatology Vol. 30, No. 1, March, 2019 https://doi.org/10.14734/PN.2019.30.1.32

\author{
Ha Shin Kim, MD', \\ Hae Sun Jeon, MD', \\ Soo Jin Han, MD', \\ Ji Hoi Kim, MD', \\ Jae Hoon Chang, MD', \\ Yun Kyu Oh, MD $D^{2,3}$, \\ Kichul Shin, $M D^{2,3}$, \\ Byoung Jae Kim, MD ${ }^{1,4}$, \\ Seung Mi Lee, MD, PhD ${ }^{1}$
}

'Department of Obstetrics and Gynecology, Seoul National University College of Medicine, Seoul;'Department of Internal Medicine, Seoul Metropolitan Government Seoul National University Boramae Medical Center, Seoul; ${ }^{3}$ Department of Internal Medicine, Seoul National University College of Medicine, Seoul; ${ }^{4}$ Department of Obstetrics and Gynecology, Seoul Metropolitan Government Seoul National University Boramae Medical Center, Seoul, Korea

Received: 24 August 2018

Revised: 9 October 2018

Accepted: 10 October 2018

Correspondence to

Seung Mi Lee, MD, PhD

Department of Obstetrics and Gynecology, Seoul National University College of Medicine, 103 Daehak-ro, Jongno-gu, Seoul 03080, Korea

Tel: $+82-2-2072-4857$

Fax: +82-2-762-3599

E-mail:smleemd@hanmail.net

Copyright@ 2019 by The Korean Society of Perinatology

This is an Open Access article distributed under the terms of the Creative Commons Attribution Non-Commercial License (http://creativecommons.org/ license/by-nc/4.0/), which permits unrestricted non-commercial use, distribution, and reproduction in any medium, provided that the original work is properly cited.

\section{Antepartum Diagnosis and Treatment of Diffuse Alveolar Hemorrhage in a Pregnant Woman with Systemic Lupus Erythematosus}

Diffuse alveolar hemorrhage (DAH) is a rare and life-threatening complication of systemic lupus erythematosus. Differential diagnosis and successful management of DAH can be problematic in pregnancy. We report a case of a 35-year-old pregnant woman who was presumptively diagnosed with DAH and treated during the antepartum period, resulting in symptomatic improvement and successful delivery after immunosuppressive treatment.

Key Words: Pregnancy complications, Lupus erythematosus, Systemic, Lupus nephritis, Hemorrhage, Lung diseases

\section{Introduction}

Diffuse alveolar hemorrhage (DAH) is a rare and life-threatening complication of systemic lupus erythematosus (SLE). Its histological feature is pulmonary capillaritis which is characterized by infiltration of neutrophils into the lung interstitium, causing structural necrosis and destruction of the capillary integrity. ${ }^{1}$ In spite of medical advances, the reported mortality of DAH is up to 50\%. For successful management, early recognition and coordination of various treatments, including cyclophosphamide, high-dose corticosteroids, and plasmapheresis, are required. ${ }^{2}$

Herein, we present a case in which DAH was suspected and treated in a pregnant woman with SLE. To our knowledge, this is the first case in which the presumptive diagnosis and treatment of DAH was performed in the antenatal period, which resulted in symptomatic improvement after immunosuppressive treatment.

\section{Case}

A 35-year-old woman, gravid 2 para 0 , visited the outpatient clinic complaining of foamy urine and swollen feet that developed 2 weeks prior at 21 weeks of gestation. Patient's medical and prior obstetric history was unremarkable. The blood pressure was within normal range $(120 / 80 \mathrm{mmHg}$ ) but the urine dipstick test suggested proteinuria with a value of 3+. Additional laboratory tests were performed, and the result of laboratory tests showed severe anemia and azotemia, and evidences of SLE. The detailed results were as follows; hemoglobin (Hb) was $5.0 \mathrm{~g} / \mathrm{dL}$, serum creatinine was $1.57 \mathrm{mg} / \mathrm{dL}, 24$-hour urinary protein excretion was $5,900 \mathrm{mg} / \mathrm{day}$, anti-nuclear antibody and anti-double stranded DNA antibody were positive, and serum complement C3 and C4 were $65.5 \mathrm{mg} / \mathrm{dL}$ (normal range, $90-180 \mathrm{mg} / \mathrm{dL}$ ) and $8.2 \mathrm{mg} / \mathrm{dL}$ (normal range, 10-40 mg/dL), respectively. Renal 
ultrasound finding was normal, and the patient decided not to undergo kidney biopsy after counseling. Antibodies against SS-A and Smith were positive and antibodies against SS-B and ribonuclear protein were negative. Lupus anticoagulant was negative. She had the period of observation on proteinuria and azotemia without any treatment for SLE.

At 26 weeks' gestation, she was admitted because of sudden dyspnea (hospitalized day 1). The chest X-ray showed cardiomegaly, bilateral pleural effusions and diffuse infiltrations suggesting pulmonary edema. Echocardiography demonstrated moderate amount of pericardial effusion but without hemodynamic significance. With the diagnosis of SLE and active nephritis, she was treated with pulse therapy of methylprednisolone $1 \mathrm{~g}$ intravenously once a day for 3 days. In addition to corticosteroid therapy, hemodialysis (plus ultrafiltration) was started to improve pulmonary edema. Soon after dyspnea improved and the corticosteroid pulse therapy was followed by maintenance dose of oral prednisolone of $1 \mathrm{mg} / \mathrm{kg} / \mathrm{day}$.

During hospitalization, fetal surveillance was performed. The fetal size was small for gestational age and the end-diastolic velocity in the umbilical artery became absent from hospitalized day 20 (28+2 weeks' gestation). As the results of nonstress test and biophysical profile were reassuring, close fetal surveillance was planned.

However, on hospitalized day 29, she developed acute aggravation of dyspnea and blood tinged sputum. Because of having severe orthopnea, she could not maintain a supine position. Laboratory test showed a sudden drop of hemoglobin (from 9.0 to $7.4 \mathrm{mg} / \mathrm{nL}$ ). Chest X-ray revealed increased consolidation of both lungs (Fig. 1A). A non-contrast computed tomography scan was performed and it revealed diffuse ground glass opacity and nodular consolidation (Fig. 1B). A presumptive diagnosis of DAH was made and methylprednisolone pulse therapy was restarted. In addition, empirical antibiotic treatment was also started, because the possibility of pneumonia could not be ruled out, in spite of absence of fever. After being informed of both fetal and her status, the patient consented to deliver the baby after the disease activity was stabilized. On hospitalized day 33 (30+1 gestation weeks), orthopnea was improved after methylprednisolone pulse therapy, and Cesarean section was performed under spinal anesthesia. A female infant weighing $970 \mathrm{~g}$ was born, and Apgar scores were 3 and 7 at one and five minutes, respectively.

After delivery, she was admitted to the intensive care unit and treated with cyclophosphamide and methylprednisolone pulse therapy. Bronchoalveolar lavage was performed and sanguineous fluid was obtained, confirming DAH. Kidney biopsy was performed on hospitalized day 44, and the pathology result was lupus nephritis International Society of Nephrology/Renal Pathology Society class III-G. On hospitalized day 45, her respiratory function deteriorated needing extracorporeal membrane oxygenation and plasmapheresis was also performed for three times. After continuous renal replacement therapy, on
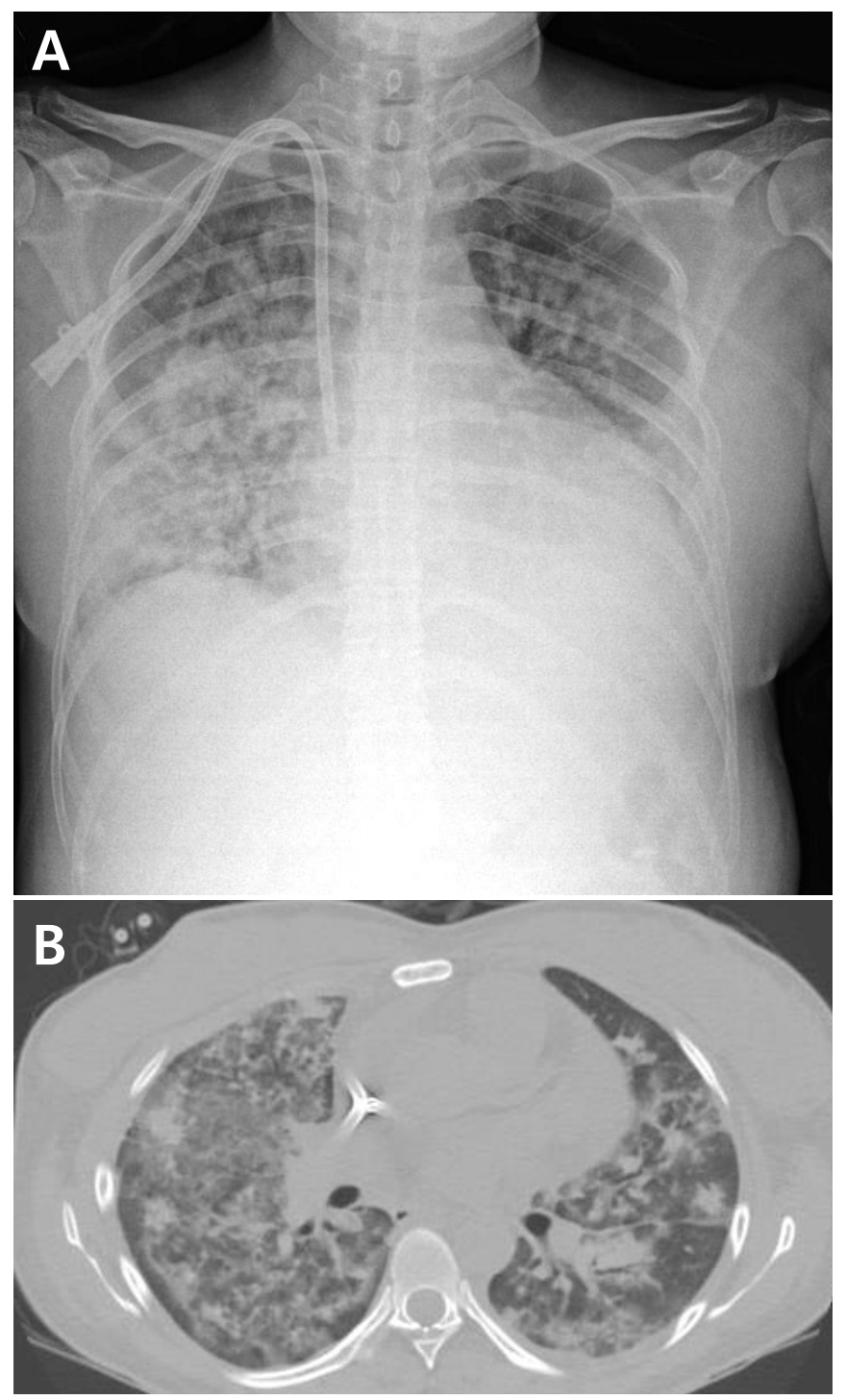

Fig. 1. (A) Chest radiograph showing diffuse consolidation. (B) Chest computed tomography scan showing diffuse ground glass opacity and nodular consolidation. 


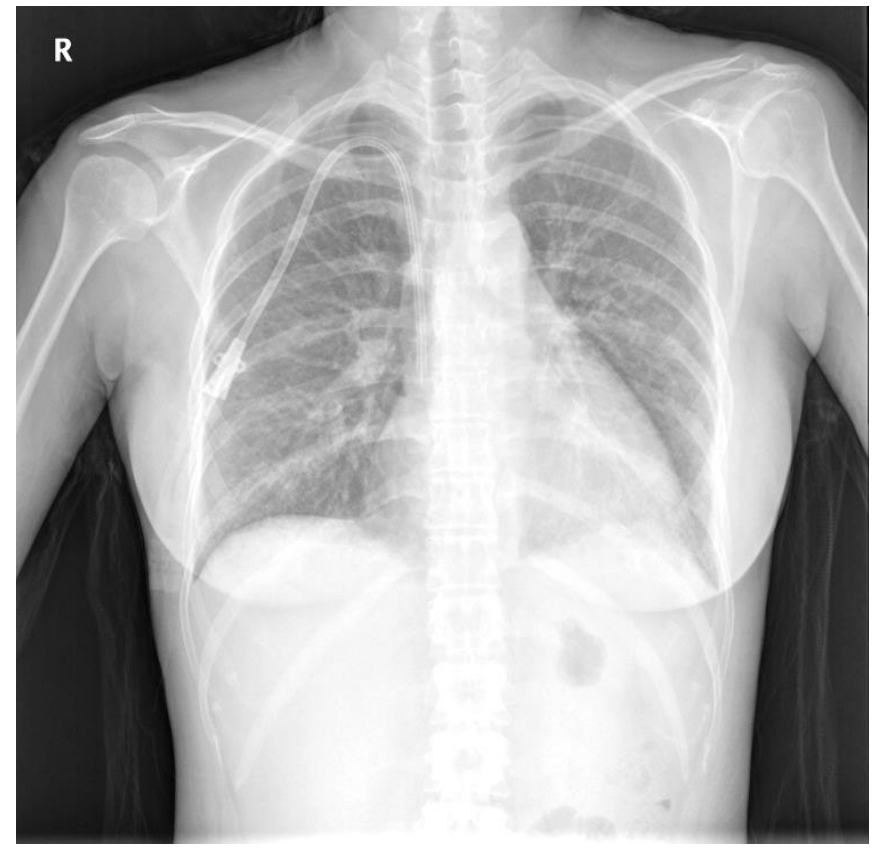

Fig. 2. Chest radiograph showing radiographic improvement.

hospitalized day 51, she was successfully extubated and recovered well, and she was discharged on hospitalized day 69. At her postpartum visit, renal function, complement and antids DNA antibody level were normalized (Fig. 2).

\section{Discussion}

SLE is a systemic autoimmune connective tissue disease. It usually occurs in women of reproductive age, and it can occur or relapse during pregnancy due to immune-endocrine changes. Aggravation of SLE occurs in about 20-30\% of pregnancies in women with SLE.

SLE can affect any organ at any stage during the course of the disease, but lung is involved in the relatively late period. The clinical manifestation of lung involvement can be various, including pleuritis, pleural effusion, parenchymal lung disease, diaphragmatic dysfunction, upper airway dysfunction, and pulmonary vascular disease such as diffuse alveolar hemorrhage (DAH). ${ }^{3,4}$

DAH is a life-threatening pulmonary complication of SLE, with the reported prevalence of 1.5 to $5.4 \%$., It usually occurs in SLE patients with active lupus nephritis or other organ involvement. The accompanying symptoms include dyspnea, cough, hemoptysis and sometimes fever. In SLE patients with sudden dyspnea, several differential diagnoses should include upper respiratory tract infection, interstitial lung disease, and rare conditions such as DAH, lupus pneumonitis, and other infections. ${ }^{3,4}$ The differential diagnosis can be problematic in pregnant women, because of the limitation in the available diagnostic modalities. In this case, the first presumptive diagnosis was DAH, based on hemoptysis, abruptly decreased hemoglobin level, and the results of chest X-ray and chest computed tomography, but antibiotics were co-administered because pneumonia could not be ruled out. The definite diagnosis needs bronchoalveolar lavage (BAL), ${ }^{5}$ but the attending physician and patient carefully decided to undergo BAL in the postpartum period.

DAH can be treated with high doses of steroids and cyclophosphamide, and plasmapheresis can be used as an adjuvant treatment. $^{7-10}$ In spite of aggressive treatment, recently reported mortality rate is still high (approximately 50\%). ${ }^{5,11}$ In pregnant women with SLE, persistently high disease activity of SLE in spite of medical treatment can be an indication of prompt delivery. However, it is not well determined whether we should decide prompt delivery in case of DAH from SLE, because it is well known that DAH can be improved with medical treatment. In this case, the patient decided to deliver the baby because of both maternal and fetal condition, which was SLE aggravation and fetal growth restriction with abnormal umbilical artery Doppler. But in terms of DAH, she experienced improvement of orthopnea by successful steroid pulse therapy, which allowed cesarean section under spinal anesthesia. To our knowledge, this is the first case in which the presumptive diagnosis and treatment of DAH was performed in the antenatal period, which resulted in symptomatic improvement and successful delivery. In the literature, there have been three case reports on DAH from pregnant women with SLE. ${ }^{12-14}$ In these cases, delivery was decided immediate after the presumptive or definite diagnosis of DAH. One of the cases is a 22-year-old pregnant woman who was presumptively diagnosed with DAH at 27 weeks of gestation and decided to start management after prompt delivery. ${ }^{12}$ Two other cases each presented with DAH at 21 weeks of gestation and 17 weeks of gestation, and both recovered after termination of the baby. ${ }^{13,14}$

Herein, we present a case of successful management of 
DAH during pregnancy. This is the first case showing that the treatment as well as presumptive diagnosis of DAH could be performed during the antenatal period. This also resulted in symptomatic improvement after immunosuppressive treatment. Though we decided to deliver because of the SLE status with poor fetal condition, this case shows that DAH could be properly managed during pregnancy. As there is no consensus whether delivery is immediately indicated in pregnant woman with DAH, further studies are needed to establish treatment strategies of DAH and timing of delivery in pregnant women with SLE.

\section{Conflict of Interest}

No potential conflict of interest relevant to this article was reported.

\section{Acknowledgments}

This research was supported by the grant 0420170550 from the SNUH research fund.

\section{References}

1) Lara AR, Schwarz MI. Diffuse alveolar hemorrhage. Chest 2010;137:1 16471.

2) Martínez-Martínez MU, Abud-Mendoza C. Diffuse alveolar hemorrhage in patients with systemic lupus erythematosus. Clinical manifestations, treatment, and prognosis. Reumatol Clin 2014;10:248-53.

3) Kamen DL, Strange C. Pulmonary manifestations of systemic lupus erythematosus. Clin Chest Med 2010;31:479-88.

4) Pego-Reigosa JM, Medeiros DA, Isenberg DA. Respiratory manifestations of systemic lupus erythematosus: old and new concepts. Best Pract Res Clin Rheumatol 2009;23:469-80.

5) Zamora MR, Warner ML, Tuder R, Schwarz MI. Diffuse alveolar hemorrhage and systemic lupus erythematosus. Clinical presentation, histology, survival, and outcome. Medicine (Baltimore) 1997;76:192-202.

6) Barile LA, Jara LJ, Medina-Rodriguez F, García-Figueroa JL, MirandaLimón JM. Pulmonary hemorrhage in systemic lupus erythematosus. Lupus 1997;6:445-8.

7) Santos-Ocampo AS, Mandell BF, Fessler BJ. Alveolar hemorrhage in systemic lupus erythematosus: presentation and management. Chest 2000;118:1083-90.

8) Mitchell K, Kaul M, Clowse ME. The management of rheumatic diseases in pregnancy. Scand J Rheumatol 2010;39:99-108.

9) Erickson RW, Franklin WA, Emlen W. Treatment of hemorrhagic lupus pneumonitis with plasmapheresis. Semin Arthritis Rheum 1994;24:11423

10) Takada K, Illei GG, Boumpas DT. Cyclophosphamide for the treatment of systemic lupus erythematosus. Lupus 2001;10:154-61.

11) Martínez-Martínez MU, Abud-Mendoza C. Predictors of mortality in diffuse alveolar haemorrhage associated with systemic lupus erythematosus. Lupus 2011;20:568-74.

12) Gaither K, Halstead K, Mason TC. Pulmonary alveolar hemorrhage in a pregnancy complicated by systemic lupus erythematosus. J Natl Med Assoc 2005;97:831-3.

13) Keane MP, Van De Ven CJ, Lynch JP 3rd, McCune WJ. Systemic lupus during pregnancy with refractory alveolar haemorrhage: recovery following termination of pregnancy. Lupus 1997;6:730-3.

14) Blitz MJ, Fleischer A. Severe maternal morbidity associated with systemic lupus erythematosus flare in the second trimester of pregnancy. Case Rep Obstet Gynecol 2018;2018:5803479. 